\title{
Avaliação da função pulmonar, força muscular periférica, independência funcional e qualidade de vida em pacientes com leucemia e linfoma durante internamento hospitalar - séries de casos
}

\author{
Evaluation of pulmonary function, peripheral muscular force, functional independence \\ and quality of life in patients with leukemia and lymphoma during hospital department- \\ case series
}

\author{
Maria Lua Motta de Moraes ${ }^{1}$, Mateus Freire², Cássio Magalhães da Silva e Silva ${ }^{3 *}$ \\ ${ }^{1}$ Acadêmica de Fisioterapia, Universidade Federal da Bahia (UFBA); ${ }^{2}$ Graduação em Fisioterapia - Universidade Federal \\ da Bahia, ; ${ }^{3}$ Docente Fisioterapia da Universidade Federal da Bahia
}

\begin{abstract}
Resumo
Introdução: Leucemias e linfomas são tipos de câncer hematológicos com importantes repercussões agravadas pelo tratamento invasivo e constantes internações. Objetivo: Avaliar a função pulmonar, força muscular periférica, independência funcional e qualidade de vida de pacientes com leucemia e linfoma hospitalizados. Resultados: A cirtometria toraco abdominal, a espirometria e a FMR apresentaram valores abaixo do predito. A força muscular global mostrou-se preservada. Os pacientes demonstraram nível de independência funcional satisfatória, a KPS revelou um valor médio de $67,6 \%$ e uma redução da qualidade de vida relacionada à saúde. Conclusão: A avaliação realizada nos pacientes internados no Complexo HUPES indicou redução na função pulmonar (cirtometria, espirometria e força muscular respiratória), independência funcional relacionada ao câncer e ao tratamento satisfatória e redução da qualidade de vida. Palavras-chave: Neoplasias. Fisioterapia. Classificação Internacional de Funcionalidade. Incapacidade e Saúde.
\end{abstract}

\section{Abstract}

Introduction: Leukemias and lymphomas are hematologic cancers with important repercussions aggravated by invasive treatment and constant admissions. Objective: To evaluate lung function, peripheral muscle strength, functional independence and quality of life of patients with hospitalized leukemia and lymphoma. Results: Abdominal thoracic cirtometry, spirometry and FMR presented values below predicted. Global muscle strength has been preserved. Patients demonstrated satisfactory functional independence, KPS revealed an average value of $67.6 \%$ and a reduction in health-related quality of life. Conclusion: The evaluation performed in patients admitted to the HUPES Complex indicated a reduction in pulmonary function (cirtometry, spirometry and respiratory muscle strength), functional independence related to cancer, and satisfactory treatment and reduction of quality of life.

Keywords: Neoplasms. Physical Therapy. International Classification of Functioning. Disability and Health.

\section{INTRODUÇÃO}

Atualmente, câncer é o nome geral dado a um grupo de doenças que têm como semelhanças o crescimento desordenado de células, que tendem a se espalhar pelos tecidos e órgãos vizinhos, ${ }^{1}$ sendo um problema de saúde pública, especialmente entre os países em desenvolvimento².

A Fisioterapia em oncologia é uma especialidade que tem como objetivo preservar, manter ou recuperar a integridade cinético-funcional de órgãos e sistemas do paciente, assim como prevenir os distúrbios causados pelo tratamento oncológico ${ }^{3}$. Porém ainda não existem muitos estudos para nortear a prática baseada em evidências.

A leucemia é uma doença maligna dos glóbulos brancos do sangue ${ }^{1}$ e tem como principal característica o acúmulo de células jovens anormais na medula óssea, pre-

Correspondente/Corresponding: *Cassio Magalhães da Silva e Silva - Instituto de Ciências da Saúde. Universidade Federal da Bahia - Departamento de Fisioterapia - End: Av. Reitor Miguel Calmon s/n, Vale do Canela, Salvador, Ba. CEP: 40.110-100. - Tel: (71) 3283-7688 - End: E-mail: cassiofisio2@yahoo.com.br judicando ou impedindo a produção de glóbulos brancos, vermelhos e plaquetas ${ }^{2}$.

Todos os invasores do nosso corpo, tais como bactérias, vírus, fungos, circulam no sistema linfático, e, quando passam pelos linfonodos, são detalhadamente examinados. A partir desta análise por células especializadas, o nosso sistema imunológico sabe exatamente contra o que está lutando e recruta um grupo especial de células, os linfócitos, que irão defender o organismo contra os agentes infecciosos. O linfoma acontece quando essas células se transformam em malignas, crescendo de forma descontrolada e "contaminando" o sistema linfático ${ }^{4}$.

A quimioterapia (QT) representa um avanço no controle do câncer e é uma das principais terapêuticas para os casos de cancer hematológico, proporcionando aumento da sobrevida e da qualidade de vida. Entretanto, os agentes citostáticos têm baixo índice terapêutico e suas ações farmacológicas podem causar eventos adversos variados, sendo os mesmos divididos em não-hematológicos, por exemplo, as toxicidades cardíacas, pulmonares, hepáti- 
cas, gastrointestinais, renais, dermatológicas, disfunções reprodutivas, alterações neurológicas, vesicais, alterações metabólicas, reações alérgicas, fadiga e em hematológicos, por exemplo, a leucopenia, anemia, trombocitopenia e a neutropenia febril ${ }^{5}$.

O tratamento oncológico é demorado e frequentemente requer hospitalização, proporcionando grande risco aos pacientes devido efeitos decorrentes desse meio 6.0 imobilismo no leito hospitalar, comum nos pacientes críticos, acomete diversos sistemas, tais como o sistema musculoesquelético, cardiorrespiratório, metabólico e neurológico, ${ }^{7}$ impactando na qualidade de vida desses pacientes.

A qualidade de vida relacionada à saude (QVRS) representa o valor atribuído à vida diante das modificações por danos na estrutura e na função do indivíduo. Conhecer a percepção do indivíduo perante a doença e conectar estes fatores às repercussões físicas do câncer faz-se importante no sentido de entender quais as suas reais necessidades e traçar melhores estratégias acerca do cuidado8.

Até então, não foram encontrados estudos que avaliassem a função pulmonar, força muscular periférica, independência funcional e qualidade de vida nos indivíduos com Leucemia e Linfoma, logo esse trabalho pode ser de fundamental importância para estudos maiores no futuro, que poderão direcionar a atuação da Fisioterapia na área da Oncologia. Assim o objetivo deste trabalho foi avaliar a função pulmonar, força muscular periférica, independência funcional e qualidade de vida de pacientes com leucemia e linfoma hospitalizados.

\section{METODOLOGIA}

Trata-se de um estudo de séries de casos, realizado em um Hospital Público Universitário. A coleta de dados ocorreu entre agosto e dezembro de 2017.

O estudo respeitou os aspectos éticos de pesquisa envolvendo seres humanos, constantes na resolução CNS 466/12, aprovado pelo Comitê de Ética em Pesquisa do Complexo Hospitalar Universitário Professor Edgar Santos - Universidade Federal da Bahia, sob parecer de número: 1.805.649. Todos os participantes leram, concordaram e assinaram o Termo de Consentimento Livre e Esclarecido. O estudo foi conduzido de acordo com a Declaração de Helsinque, revisada em 2008.

A amostra de conveniência foi composta pelos pacientes com leucemia e linfoma admitidos pela Enfermaria de um hospital público universitário durante o período da coleta de dados, de acordo com os critérios do estudo. Critérios de inclusão: ter idade igual ou superior a 18 anos, ser portador de leucemia ou linfoma, estar admitido na enfermaria oncológica. Critérios de exclusão: pacientes com infecções não controladas de qualquer natureza, paciente com dificuldade de compreensão cognitiva, incapacidade de responder informações contidas nos questionários aplicados na avaliação e reavaliação, pacientes instáveis hemodinamicamente e em isolamento de contato e/ou aéreo.

Os procedimentos de coleta de dados foram realizados através de uma Ficha de Avaliação elaborada pelo autor, contendo informações sociodemográficas e clínicas do paciente. Nesta mesma ficha foram registrados os resultados da avaliação física. Os questionários e escalas utilizados estavam anexados a esta ficha. Todas as avaliações foram realizadas por apenas um avaliador e duraram cerca de 40 minutos.

A força muscular respiratória (FMR) foi avaliada através da Manovacuometria, obtendo-se assim a pressão inspiratória máxima (PIM) e pressão expiratória máxima (PEM). Para realização deste teste, foram seguidas as normas da American Thoracic Society/European Respiratory Society e as equações dos valores previstos utilizadas foram propostas por Neder et al. . $^{910} \mathrm{O}$ bocal adaptado ao Manovacuômetro (Wika 611.10) possui um orifício de 2 milímetros, evitando assim a contração dos músculos bucinadores por aumento da pressão intraoral que pudesse interferir nos resultados.

A função pulmonar foi avaliada através da Espirometria, sendo obtidos o volume expiratório forçado no primeiro segundo $\left(V_{E F}\right)$ e a capacidade vital forçada (CVF), através do espirômetro Micro Medical. A partir destes dados, foi calculada a relação $V E F_{1} / C V F$. Para a realização deste teste foram seguidas as normas da American Thoracic Society/ European Respiratory Society ${ }^{9}$. Os valores de referência utilizados foram baseados na tabela que vem anexada ao espirômetro.

A força muscular periférica (FMP) foi avaliada através da escala do Medical Research Council, instrumento para avaliação da força muscular de membros superiores (MMSS) e membros inferiores (MMII). Os movimentos avaliados foram: abdução do ombro, flexão do cotovelo, extensão do punho, flexão do quadril, extensão do joelho dorsiflexão do tornozelo. ${ }^{11}$

O nível de independência funcional foi avaliado através da Medida de Independência Funcional (MIF). A MIF é um questionário validado e amplamente utilizado no Brasil, que quantifica o grau de independência ou solicitação de cuidados de terceiros que o indivíduo possui para realização de AVD's, envolvendo aspectos motores e cognitivos ${ }^{12,13}$ Seus seis domínios contêm atividades e funções específicas da vida diária, cada uma podendo variar em uma pontuação de 1 (dependência total) a 7 (independência total). A dependência pode envolver a necessidade de outros indivíduos ou de dispositivos auxiliares.

O nível funcional e a evolução do paciente com o tratamento foram avaliados pela escala de Desempenho de Karnofsky (KPS). A KPS descreve e classifica o perfil da população com câncer em três grupos, conforme o seu grau de aptidão para trabalhar, exercer atividades cotidianas e necessidades de cuidados, contendo: capaz de realizar atividade normal, não precisa de cuidado especial ( 80 a 100\%); incapaz de trabalhar, capaz de viver em casa e cuidar da maioria das necessidades pessoais, mas necessita de uma quantidade variável de assistência (50 a 70\%); incapaz de cuidar de si mesmo requer o equivalente de cuidado institucional ou hospitalar, a doença pode estar progredindo rapidamente (0 a 40\%). Quanto menor a pontuação, pior a expectativa de recuperação da enfermidade ou retorno às atividades normais ${ }^{14,15}$.

Para avaliar a qualidade de vida relacionada à saúde (QVRS) foi utilizada a escala Funcional Assessment of Can- 
cer Therapy (FACT-G) Versão 4, desenvolvida pelo sistema Functional Assessment of Chronic Illness Therapy (FACIT) e que aborda 27 itens em 4 domínios: bem-estar físico, bem-estar social/familiar, bem-estar emocional e bem-estar funcional. Cada um destes domínios possui pontuação máxima de $28,28,24$ e 28 pontos respectivamente. O escore total varia de 0-108 pontos, sendo este a soma dos resultados de cada domínio. Esta escala é considerada apropriada para todos os tipos de câncer e validada para a população brasileira em $2011^{16}$. Foi feita uma regra de 3 para mostrar em porcentagem a QVRS destes pacientes.

\section{RESULTADOS}

O estudo foi composto por uma amostra de conveniência de sete pacientes sendo quatro com leucemia e três com linfoma, variando entre 22 e 66 anos de idade. Destes sete, quatro pacientes são do sexo masculino e três pacientes do sexo feminino. As características sócias demográficas estão na tabela 1 .

Tabela 1 - Características sócio-demográficas e clínicas dos pacientes com leucemia e linfoma internados na enfermaria 1B do Complexo Hupes, Salvador-Ba, 2017.

\begin{tabular}{|c|c|c|c|}
\hline Paciente / Sexo/ Idade (anos) & Estado Civil/ Altura (cm) & Tipo de câncer / Ciclo de QT & $\begin{array}{c}\text { Doenças pulmonares prévias / } \\
\text { Tabagismo prévio }\end{array}$ \\
\hline 1 / Masculino / 23 & Solteiro (a)/ 172 & Linfoma / 20 & Nega \\
\hline 2 / Masculino / 53 & Casado (a)/ 169 & Linfoma / 20 & Nega \\
\hline 3 / Masculino / 35 & Casado (a)/ 181 & Leucemia / 5음 & Nega \\
\hline 4 / Feminino / 22 & Solteiro (a)/ 159 & Leucemia / 3음 & Nega \\
\hline 5 / Feminino / 31 & Casado (a) / 162 & Leucemia / $4^{\circ}$ & Nega \\
\hline $6 /$ Feminino / 54 & Casado (a) / 163 & Linfoma / 1은 & Nega \\
\hline 7 / Masculino / 66 & Casado (a) / 157 & Leucemia / 10 & Nega \\
\hline
\end{tabular}

QT: quimioterapia; cm: centímetros.

Fonte: Autoria Própria.

A FMR da amostra apresentou-se abaixo do valor predito para ambos os sexos, sendo os pacientes 2 e 3 os únicos a não apresentarem distúrbio na FMR.

Tabela 2 - Avaliação da força muscular respiratória dos pacientes com leucemia e linfoma internados na enfermaria $1 B$ do complexo Hupes, Salvador - Ba 2017.

\begin{tabular}{|c|c|c|c|c|c|}
\hline \multirow{2}{*}{$\begin{array}{l}\text { Paciente } \\
1\end{array}$} & \multicolumn{5}{|c|}{ Força Muscular Respiratória $\left(\mathrm{cmH}_{2} \mathrm{O}\right)$} \\
\hline & & 10 Dia & 5 Dia & 10 Dia & Predito \\
\hline & PIM & -40 & -35 & -30 & $-136,9$ \\
\hline & PEM & 40 & 35 & 35 & 146,67 \\
\hline \multirow[t]{3}{*}{2} & & 10 Dia & 5ㅇia & 10 Dia & Predito \\
\hline & PIM & -80 & -100 & -100 & $-112,9$ \\
\hline & PEM & 50 & 60 & 60 & 122,37 \\
\hline \multirow[t]{3}{*}{3} & & 10 Dia & 5o Dia & 10 Dia & Predito \\
\hline & PIM & -100 & -70 & -70 & $-127,3$ \\
\hline & PEM & 80 & 50 & 50 & 136,95 \\
\hline \multirow[t]{3}{*}{4} & & 10 Dia & 5o Dia & 10 Dia & Predito \\
\hline & PIM & -35 & -20 & -20 & $-99,62$ \\
\hline & PEM & 20 & 20 & 20 & 102,18 \\
\hline \multirow[t]{3}{*}{5} & & 10 Dia & 5 Dia & 10 Dia & Predito \\
\hline & PIM & -50 & -60 & -60 & 125,85 \\
\hline & PEM & 40 & 50 & 40 & 98,27 \\
\hline \multirow[t]{3}{*}{6} & & 10 Dia & 5o Dia & 10 Dia & Predito \\
\hline & PIM & -30 & -40 & -50 & 65,05 \\
\hline & PEM & 30 & 40 & 30 & 82,63 \\
\hline \multirow[t]{3}{*}{7} & & 1임 & 5으 Dia & 10 Dia & Predito \\
\hline & PIM & -40 & -30 & -50 & 150,3 \\
\hline & PEM & 50 & 60 & 50 & 100,58 \\
\hline
\end{tabular}

PIM: pressão inspiratória máxima; PEM: pressão expiratória máxima; $\mathrm{CmH}_{2} \mathrm{O}$ : centímetros de água.
Na Espirometria, a amostra apresentou valores abaixo do predito tanto para $V_{E F}^{1}$ e CVF quanto para a relação entre eles $\left(\mathrm{VEF}_{1} / \mathrm{CVF}\right)$.

Tabela 3 - Avaliação da função pulmonar dos pacientes com leucemia e linfoma internadosna enfermaria $1 B$ do complexo Hupes, Salvador - Ba 2017.

\begin{tabular}{|c|c|c|c|c|c|}
\hline \multirow{2}{*}{$\begin{array}{l}\text { Paciente } \\
1\end{array}$} & \multicolumn{5}{|c|}{ Espirometria } \\
\hline & & 19Dia & 5Dia & 10Dia & Predito \\
\hline & $\operatorname{VEF}_{1}$ (L) & 2,58 & 2,25 & 2,61 & 4,24 \\
\hline & $\operatorname{CVF}^{1}(\mathrm{~L})$ & 3,38 & 3,37 & 3,60 & 5,05 \\
\hline & $\operatorname{VEF}_{1} / \mathrm{CVF}(\%)$ & 76 & 66 & 72 & 83 \\
\hline \multirow[t]{4}{*}{2} & & 19Dia & 5Dia & 10Dia & Predito \\
\hline & $\operatorname{VEF}_{1}(\mathrm{~L})$ & 2,14 & 2,27 & 2,27 & 3,03 \\
\hline & $\operatorname{CVF}^{1}(\mathrm{~L})$ & 2,38 & 2,50 & 2,50 & 3,73 \\
\hline & $\operatorname{VEF}_{1} / \mathrm{CVF}(\%)$ & 90 & 91 & 91 & 81 \\
\hline \multirow[t]{4}{*}{3} & & 19Dia & 5Dia & 10Dia & Predito \\
\hline & $\operatorname{VEF}_{1}(\mathrm{~L})$ & 1,79 & 2,33 & 2,30 & 4,62 \\
\hline & $\operatorname{CVF}^{1}(\mathrm{~L})$ & 3,52 & 4,21 & 4,20 & 5,61 \\
\hline & $\operatorname{VEF}_{1} / \mathrm{CVF}(\%)$ & 50 & 55 & 54,6 & 82 \\
\hline \multirow[t]{4}{*}{4} & & 1Dia & 5Dia & 10 Dia & Predito \\
\hline & $\operatorname{VEF}_{1}(\mathrm{~L})$ & 1,88 & 1,87 & 1,80 & 2,93 \\
\hline & $\operatorname{CVF}^{1}(\mathrm{~L})$ & 3,70 & 2,67 & 2,65 & 3,37 \\
\hline & $\operatorname{VEF}_{1} / \mathrm{CVF}(\%)$ & 51,8 & 70 & 68 & 86 \\
\hline \multirow[t]{4}{*}{5} & & 1ํDia & 5Dia & 10Dia & Predito \\
\hline & $\operatorname{VEF}_{1}(\mathrm{~L})$ & 1,8 & 3,2 & 2,5 & 2,91 \\
\hline & $\operatorname{CVF}^{1}(\mathrm{~L})$ & 2,86 & 3,23 & 2,98 & 3,43 \\
\hline & $\operatorname{VEF}_{1} / \mathrm{CVF}(\%)$ & 68 & 77 & 91 & 85 \\
\hline \multirow[t]{4}{*}{6} & & 1ํDia & 5Dia & 10Dia & Predito \\
\hline & $\operatorname{VEF}_{1}(\mathrm{~L})$ & 1,59 & 1,68 & 1,57 & 2,53 \\
\hline & $\operatorname{CVF}^{1}(\mathrm{~L})$ & 2,02 & 2,28 & 2,19 & 3,09 \\
\hline & $\operatorname{VEF}_{1} / \mathrm{CVF}(\%)$ & 78 & 73 & 71 & 82 \\
\hline \multirow[t]{4}{*}{7} & & 1Dia & 5Dia & 10 Dia & Predito \\
\hline & $\operatorname{VEF}_{1}(\mathrm{~L})$ & 0,91 & 0,92 & 0,85 & 1,91 \\
\hline & $\operatorname{CVF}^{1}(\mathrm{~L})$ & 1,48 & 1,46 & 1,58 & 2,42 \\
\hline & VEF $_{1} /$ CVF (\%) & 62 & 62 & 53 & 79 \\
\hline
\end{tabular}

L: litros; $\mathrm{VEF}_{1}$ : volume expiratório forçado no primeiro segundo; $\mathrm{CVF}$ : capacidade vital forçada; $\mathrm{VEF}_{1} / \mathrm{CVF}$ : relação entre volume expiratório forçado no primeiro segundo e capacidade vital forçada.

Fonte: Autoria Própria

Rev. Ciênc. Méd. Biol., Salvador, v. 17, n. 2, p. 194-198, mai./jun. 2018 
Na tabela 4 segue as informações sobre a medida de força muscular periférica e da qualidade de vida relacionada à saúde de cada paciente. Enquanto na tabela 5 estão dispostas a independência e capacidade funcional dos pacientes.

Tabela 4 - Avaliação da força muscular periférica e qualidade de vida relacionada à saúde dos pacientes com leucemia e linfoma internados na enfermaria $1 B$ do Complexo Hupes, Salvador - BA, 2017.

\begin{tabular}{|c|c|c|c|c|c|c|}
\hline \multirow[t]{2}{*}{ Pacientes } & \multicolumn{3}{|c|}{$\begin{array}{l}\text { FORÇA MUSCULAR } \\
\text { PERIFÉRICA }\end{array}$} & \multicolumn{3}{|c|}{$\begin{array}{l}\text { QUALIDADE DE VIDA } \\
\text { RELACIONADA À SAÚDE }\end{array}$} \\
\hline & $10 \mathrm{dia}$ & 5o dia & 10 o dia & $\begin{array}{l}\text { 10 dia } \\
(\%)\end{array}$ & $\begin{array}{l}\text { 5o dia } \\
(\%)\end{array}$ & $\begin{array}{c}10 \text { o dia } \\
(\%)\end{array}$ \\
\hline 1 & 60 & 60 & 60 & 49 & 55 & 54 \\
\hline 2 & 60 & 60 & 60 & 53 & 53 & 52 \\
\hline 3 & 60 & 60 & 60 & 47 & 49 & 49 \\
\hline 4 & 55 & 54 & 54 & 55 & 58 & 57 \\
\hline 5 & 55 & 54 & 54 & 72 & 78 & 68 \\
\hline 6 & 55 & 54 & 54 & 76 & 77 & 79 \\
\hline 7 & 60 & 59 & 59 & 73 & 72 & 75 \\
\hline
\end{tabular}

Fonte: Autoria Própria

Tabela 5 - Pontuação da independência e capacidade funcional dos pacientes com leucemia e linfoma na enfermaria $1 B$ do complexo Hupes, Salvador - Ba 2017.

\begin{tabular}{|c|c|c|c|c|c|c|}
\hline \multirow[t]{2}{*}{ Paciente } & \multicolumn{3}{|c|}{$\begin{array}{l}\text { MEDIDA DE } \\
\text { INDEPENDÊNCIA } \\
\text { FUNCIONAL }\end{array}$} & \multicolumn{3}{|c|}{ KARNOFSKY } \\
\hline & 10 dia & 5o dia & $10 \%$ dia & 10 dia & 5o dia & $10 \%$ dia \\
\hline 1 & 126 & 126 & 126 & 60 & 60 & 60 \\
\hline 2 & 122 & 121 & 121 & 80 & 90 & 90 \\
\hline 3 & 126 & 126 & 126 & 70 & 90 & 90 \\
\hline 4 & 125 & 124 & 124 & 60 & 60 & 60 \\
\hline 5 & 122 & 121 & 121 & 60 & 60 & 60 \\
\hline 6 & 122 & 121 & 121 & 60 & 60 & 60 \\
\hline 7 & 121 & 121 & 121 & 70 & 60 & 60 \\
\hline
\end{tabular}

Fonte: Autoria Própria

\section{DISCUSSÃO}

A Fisioterapia se utiliza de métodos e técnicas que atuam tanto na melhora da sintomatologia quanto na prevenção e qualidade de vida. O tratamento fisioterápico é imprescindível para qualquer indivíduo cuja atividade diária esteja comprometida. Nos processos de doença, contribiu na redução de quadros dolorosos e evita possíveis complicações após cirurgias ou longos períodos de imobilização ${ }^{17}$.

Os aspectos cruciais obtidos na presente pesquisa foram à redução na função pulmonar, força muscular respiratória e qualidade de vida relacionada à saúde. Por outro lado, a força muscular periférica, a medida de independência e capacidade funcional encontra-se dentro dos parâmetros funcionais de normalidade.

Pacientes com tempo de internação prolongado demonstram prejuízos na ventilação pulmonar devido à diminuição da força muscular respiratória. Ao avaliar a PIM, podem ser identificados: fraqueza muscular respiratória (PIM entre -70 e $-46 \mathrm{cmH} 2 \mathrm{O}$ ), fadiga muscular respiratória ( $\mathrm{PIM}$ entre $-44 \mathrm{e}-25 \mathrm{cmH} 2 \mathrm{O}$ ) e falência muscular respiratória (PIM igual a $-20 \mathrm{cmH} 2 \mathrm{O}$ ). Diante do exposto, o paciente 1 apresentou fadiga muscular respiratória, o paciente 4 sugeriu falência muscular respiratória e os pacientes 5, 6 e 7 demonstram fraqueza muscular respiratória no décimo dia de internamento ${ }^{18}$.

De acordo com Pereira e colaboradores a maioria dos pacientes submetidos à avaliação da capacidade funcional global apresenta um estado funcional prévio à hospitalização, uma vez que já se encontram expostos a diversos fatores de risco desde a primeira semana de internação. Eles estimam que parte significativa do declínio funcional ocorra em poucos dias antes da internação. O que explica os valores abaixo do predito no primeiro dia da avaliação referente à Espirometria ${ }^{19}$. Nota-se redução no VEF ${ }_{1}$ e CVF dos pacientes durante o internamento do primeiro ao décimo dia de internamento. Esses dados corroboram com os achados de outros estudos que demonstram que o tempo de internamento hospitalar está associado a uma diminuição dos volumes pulmonares, atrelados ao posicionamento em decúbito dorsal e imobilidade no leito ${ }^{20,21}$.

No presente estudo observou-se uma redução da relação $\operatorname{VEF}_{1} / \mathrm{CVF}$ na maior parte da amostra. Não foram detectados sintomas respiratórios na amostra e foi identificado distúrbio obstrutivo nos pacientes 3, 4 e 7 baseando-se na espirometria, apesar dos mesmos negarem tabagismo e doenças pulmonares prévias. Vione e colaboradores avaliaram a função pulmonar de pacientes submetidos à quimioterapia a partir do terceiro ciclo; distúrbios pulmonares dos tipos obstrutivo, restritivo e misto em pacientes com leucemia, linfoma e câncer de mama foram encontrados ${ }^{5}$. Os pacientes 4 e 5 apresentaram uma melhora na relação VEF $/$ CVF. Não foram encontrados artigos na literatura que justifiquem tal melhora. Apesar de os pacientes realizarem de um a dois atendimentos de Fisioterapia diários, não há comprovações científicas de melhora na função pulmonar após treinamento de exercício físico ${ }^{22}$.

A força muscular global da amostra permaneceu preservada, com valores médios totais na escala MRC de 59 para o sexo masculino e de 54 para o sexo feminino. Estes dados não corroboram com a literatura, pois a imobilização do sistema musculoesquelético gera alterações em todos os tipos de tecidos envolvidos, nos músculos, nas fibras de colágeno, na junção miotendinosa, ligamentos e tecido conjuntivo. ${ }^{23}$ Demonstrando que o acompanhamento fisioterapêutico na unidade oncohematológica traz bons resultados aos pacientes.

Segundo os critérios avaliados pela escala MIF, os pacientes demonstraram nível de independência funcional satisfatória. A média da pontuação total foi de 123 pontos, sendo 126 a soma máxima que se pode obter na escala. Os valores obtidos encontram-se dentro da pontuação que representa independência completa nas principais atividades do dia a dia. O declínio funcional é uma condição inerente à trajetória da maioria das doenças que ameaçam a continuidade da vida e ocorrem em meio a um processo de profundas alterações físicas e emocionais. As condições em que este declínio se revela são sempre heterogêneas ${ }^{24}$. Elas diferem de acordo com a patologia, os tratamentos utilizados, as comorbidades e a condição social, familiar e subjetiva de cada paciente ${ }^{23}$.

A KPS revelou um valor médio de 67 pontos, demonstrando apresentar alguns sintomas, realizar atividades normais com necessidade de ajuda, com esforço e ser incapaz de trabalhar ativamente.

Santos e colaboradores afirmam que o internamento hospitalar se associa a diminuição da QVRS $^{25}$. O câncer e seu tratamento afetam de algum modo a QVRS dos pacientes ${ }^{26}$. Esses resultados corroboram com os achados da amostra que apresentou uma redução da QVRS seja pelo internamento hospitalar, pelo processo de adoecimento ou pelo tratamento.

Considera-se como limitação deste estudo o número reduzido da amostra e a discriminação entre os tipos de leucemias e linfomas.

\section{CONCLUSÃO}

Nesta série de casos, observou-se que os pacientes com leucemia e linfoma internados apresentaram redução na função pulmonar ( espirometria e força muscular respiratória), uma independência funcional relacionada ao câncer e ao tratamento satisfatória e redução da qualidade de vida. Tais achados podem contribuir para identificação das necessidades e definição das prioridades de tratamento da população avaliada. O reduzido tamanho amostral do estudo limita a possibilidade de generalização dos desfechos, portanto sugere-se a realização de estudos maiores com a mesma temática.

\section{REFERÊNCIAS}

1. INSTITUTO NACIONAL DE CÂNCER JOSÉ ALENCAR GOMES DA SILVA. $A B C$ do câncer: abordagens básicas para o controle do câncer. Rev. e 
Atual, [s.I], n. 2, p. 17-34, 2012.

2. BRASIL. Ministério da Saúde. Instituto Nacional de Câncer. Estimativas 2016: incidência de câncer no Brasil. Rio de Janeiro: Ministério da Saúde, 2015.

3. INSTITUTO NACIONAL DE CÂNCER JOSÉ ALENCAR GOMES DA SILVA. Fisioterapia. Rio de Janeiro, 2018.

4. ASSOCIAÇÃO BRASILEIRA DE LINFOMA E LEUCEMIA. Linfoma. São Paulo, 2016.

5. VIONE, C. H. et al. Avaliação da função pulmonar em pacientes com câncer submetidos à quimioterapia. Rev. epidemiol controle infecç., Santa Cruz do Sul, v.6, n.1, p.1-11, 2016.

6. RODRIGUES, F. S. S.; POLIDORI, M.M. Enfrentamento e resiliência de pacientes em tratamento quimioterápico e seus familiares. Rev. bras. cancerol., Rio de Janeiro, v. 58, n. 4, p. 19-627, 2012.

7. CALLES, A. C. N. et al. O impacto da hospitalização na funcionalidade e na força muscular após internamento em unidade de terapia intensiva. Interfaces Científicas - Saúde e Ambiente, Aracaju, v.5, n.3, p. 67-76, jun. 2017.

8. FREIRE, M.E.M.et al. Qualidade de vida relacionada à saúde de pacientes com câncer avançado: uma revisão integrativa. Rev. Esc Enferm. USP, São Paulo, v. 48, n.2, p. 357-367, 2014.

9. AMERICAN THORACIC SOCIETY. EUROPEAN RESPIRATORY SOCIETY. ATS/ERS Statement on respiratory muscle testing. Am. j. respir. crit. care med., New York, v. 166, n. 4, p. 518-624, 2002.

10. NEDER, J.A. et al. Reference values for lung function tests. II. Maximal respiratory pressures and voluntary ventilation. Braz j. med. biol. res., Ribeirão Preto, v. 32, n.6, p.719-727, June 1999.

11. JONGHE, B.et al. Paresis acquired in the intensive care unit: a prospective multicenter study. Univ of Colorado Health Science Ctr., [s.I], v. 288, n.22, p. 2859-2867, Dec. 2002

12. RIBERTO, M. et al. Validação da versão brasileira da medida de independência funcional. Acta Fisiatr., São Paulo, v.11, n.2, p.72-76, Ago. 2004.

13. KIDD, D. et al. The functional independence measure: a comparative validity and reliability study. Disabil Rehabil., London, v.17, n.1, p.10-14, Jan. 1995.

14. YATES, J.W.; CHALMER, B.; MCKEGNEY, F.P. Evaluation of patients with advanced cancer using the karnofsky performance status. Cancer, [s.l], v. 45, n. 8, p.2220-2224. Apr. 1980.

15. SCHAG, C.C.; HEINRICH R.L.; GANZ P. A. Karnofsky. Performance status revisited: reliability, validity, and guidelines. J. Clin. Oncol., New York, v.2, n.3, p.187-193, Mar 1984.

16. PEREIRA, F.M.P.; SANTOS, C.S.V.B. Estudo de adaptação cultural e validação da functional assessment of cancer therapy-general em cuidados paliativos. Rev Enf Ref., [s.I], v.série III, n.5, p.45-54. dez. 2011.

17. FARIA, L. As práticas do cuidar na oncologia: a experiência da fisioetrapia em pacientes com câncer de mama. Ciências, Saúde - Manguinhos, Rio de Janeiro, v. 17, p. 69-87, Jul. 2010.

18. GONÇALVES, M. P. et al. Avaliação da força muscular inspiratória e expiratória em idosas praticantes de atividade física e sedentárias. $\mathbf{R}$. Bras. Ci e Mov. v.14, n.1, p.37-44, jan. 2006.

19. PEREIRA, E.E.B. et al. Funcionalidade global de idosos hospitalizados. Rev. bras. geriatr. gerontol., Rio de Janeiro, v.17, n.1, p.165-176, 2014.

20. PAIVA, D. N. et al. Avaliação da força de preensão palmar e dos volumes pulmonares de pacientes hospitalizados por condições não cirúrgicas. Sci. med., Porto Alegre, v. 24, n. 1, p. 61-67, 2014.

21. MORSCHEL G. R. et al. Efeitos de um programa domiciliar de exercícios físicos na capacidade funcional, na força muscular ventilatória e na qualidade de vida de portadores de Doença Pulmonar Obstrutiva Crônica. 2011,3, XII Salão de Iniciação Científica - PUCRS, 2011.

22. SILVA, B. C. A. et al. Avaliação da funcionalidade respiratória em pacientes com tempo prolongado de internação hospitalar. J. Health Sci. Inst., Cuiabá, v.32, n.4, p.433-438, 2014.

23. MARCUCCI, F.C.I. O papel da fisioterapia nos cuidados paliativos a pacientes com câncer. Rev. bras. cancerol., Rio de Janeiro, v.51, n.1, p.67-77, 2005.

24. JEYASINGAM, L. et al. A prospective study of unmet activity of daily living needs in palliative care inpatients. Aust. occup. ther. j., Melbourne, v. 55 , n. 4, p. 266-272, Dec. 2008

25. SANTOS, G.; SOUSA, L. Qualidade de vida em pessoas idosas hospitalizadas: comparação da admissão com a alta do internamento. Revista Kairós-Gerontologia, São Paulo, v.16, n.2, p.07-25, mar 2013.

26. NICOLUSSI A.C. et al. Qualidade de vida relacionada à saúde de pacientes com câncer em quimioterapia. Rev Rene, [s.I], v.15, n.1, p.132-140, jan./fev. 2014. 